\title{
Aortic valve repair for quadricuspid aortic valve associated with aortic regurgitation and ascending aortic aneurysm
}

\author{
Kozo Naito, MD, Hitoshi Ohteki, MD, Junji Yunoki, MD, Kazuhiro Hisajima, MD,
} Hisashi Sato, MD, and Yasushi Narita, CE, MS, Saga, Japan



Yunoki, Naito, Ohteki, Narita (left to right)

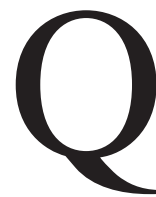

uadricuspid aortic valve is a rare congenital anomaly. Most cases have been discovered as an incidental finding at aortic valve surgery or at autopsy. With advances in echocardiography, more cases now being discovered preoperatively. Valvular insufficiency is the most frequent hemodynamic abnormality in patients with a quadricuspid aortic valve. Most patients require aortic valve replacement, and only a few cases of surgical aortic valve repair have been reported. ${ }^{1,2}$ We report a case of quadricuspid aortic valve associated with aortic regurgitation and ascending aortic aneurysm. Aortic valve repair and ascending aorta replacement with plication of the sinotubular junction were performed successfully.

\section{Clinical Summary}

A 70-year-old-woman was noted during a routine medical examination to have a diastolic murmur and was referred to our hospital for further assessment. The chest radiograph showed moderate cardiomegaly, with a cardiothoracic ratio of 0.67 . Transthoracic echocardiography demonstrated severe aortic regurgitation and quadricuspid aortic valves. Chest computed tomography showed an ascending aortic aneurysm (45 $\mathrm{mm}$ in diameter) and dilatation of the sinotubular junction ( $34 \mathrm{~mm}$ in diameter). Transesophageal echocardiography confirmed a quadricuspid valve with three equal cusps and one small cusp (type B in the Hurwitz classification ${ }^{3}$ ). The accessory cusp was located between the right and noncoronary cusps. There was no evidence of any other congenital anomaly.

During surgery in May 2003, the aortic arch was cannulated, and during normothermic cardiopulmonary bypass the heart was arrested with cold cardioplegic solution. Intraoperative assessment of aortic valves was performed with a videoscope (Olympus Optical Co, Ltd, Tokyo, Japan) through the ascending aorta with the infusion of crystalloid cardioplegic solution (Figure 1). The cusps did not coaptate in the midline and had an obvious regurgitation in the central area. The four commissures were well developed, and the cusps were slightly thickened. The left and right coronary

\footnotetext{
From the Department of Cardiovascular Surgery, Saga Prefectural Hospital, Koseikan, Saga, Japan.

Received for publication March 17, 2004; accepted for publication March 30, 2004.

Address for reprints: Kozo Naito, MD, Department of Cardiovascular Surgery, Saga Prefectural Hospital, Koseikan, 1-12-9 Mizugae, Saga 8408571, Japan (E-mail: kozonaito@ hotmail.com).

J Thorac Cardiovasc Surg 2004;128:759-60

$0022-5223 / \$ 30.00$

Copyright (C) 2004 by The American Association for Thoracic Surgery

doi:10.1016/j.jtcvs.2004.03.038
}

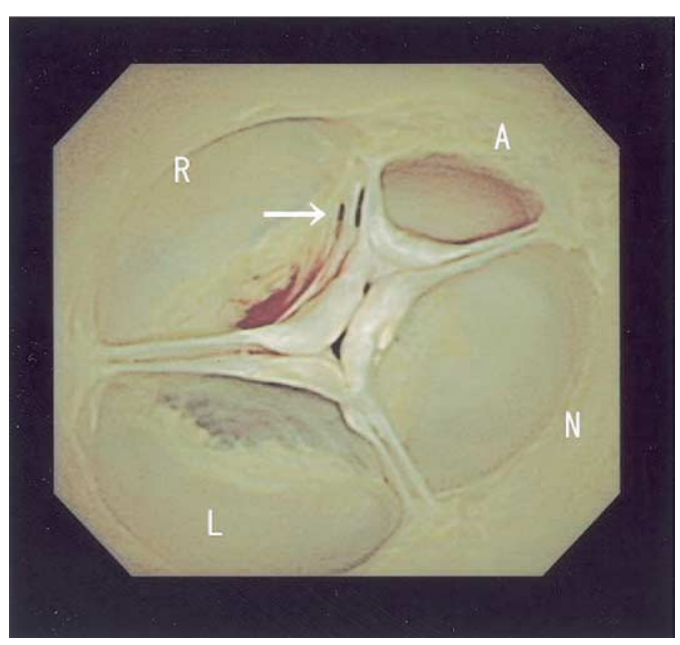

Figure 1. Preoperative videoscopic view of quadricuspid aortic valve with infusion of crystalloid cardioplegic solution. Arrow indicates fenestration (fibrous band). $\boldsymbol{R}$, Right coronary cusp; $\boldsymbol{A}$, accessory cusp; $N$, noncoronary cusp; $L$, left coronary cusp.

arteries appeared normal. Two fenestrations were seen in the right coronary cusp.

Aortic valve repair was performed by suturing together the commissure between the right coronary cusp and accessory cusp with 5-0 polypropylene suture, thus converting the quadricuspid valve into a tricuspid valve (Figure 2). Furthermore, plication of the sinotubular junction with eight mattress sutures and replacement of the ascending aorta with a 24-mm vascular graft (Hemashield; Boston Scientific Corporation, Meadox Division, Boston, Mass) were performed. Postoperative transesophageal echocardiography showed good coaptation of the repaired aortic valves with trivial aortic regurgitation (Figure 3, A) compared with the preoperative study (Figure $3, B$ ). The postoperative course was uneventful, and the patient is doing well 10 months after surgery.

\section{Discussion}

The extreme rarity of quadricuspid aortic valve has been documented both in large autopsy series and echocardiography studies, with an estimated incidence of between $0.008 \%$ and $0.013 \%{ }^{4}$ Among patients undergoing aortic valve replacement, the incidence ranges from $0.55 \%$ to $1.46 \%$. $^{2}$ Hurwitz and Roberts ${ }^{3}$ classified the quadricuspid valve into seven types on the basis of the relative size of the four cusps. The most common type consists of three equally sized cusps and one smaller cusp (type B). Because the location of the accessory cusp is not specified in this classifi- 


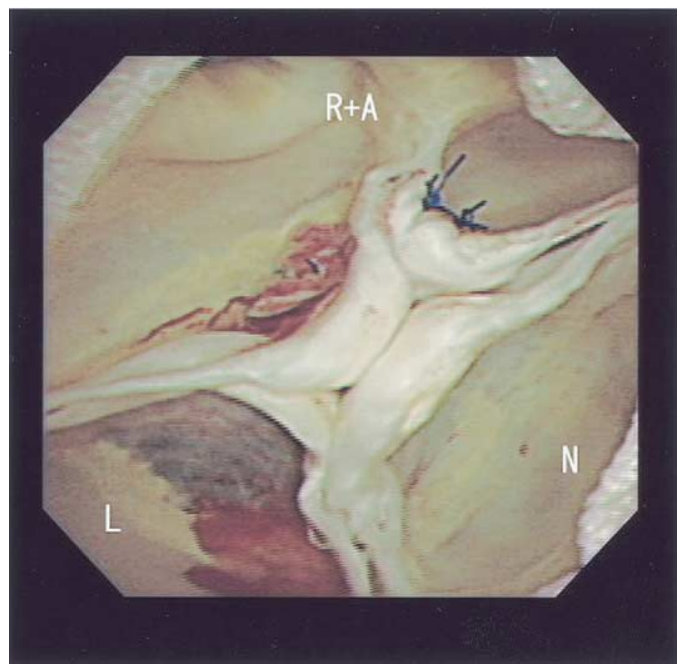

Figure 2. Postoperative videoscopic view of repaired aortic valve converted into tricuspid valve with 5-0 polypropylene sutures. $R+A$, Repaired right coronary cusp and accessory cusp; $N$, noncoronary cusp; $L$, left coronary cusp.

cation, Nakamura and colleagues ${ }^{5}$ further classified it into four variations. The most common is type II, in which the accessory cusp is located between the right coronary cusp and noncoronary cusp. Nakamura and colleagues ${ }^{5}$ analyzed the correlation between the accessory cusp position and the occurrence of aortic regurgitation and found that position did not affect the incidence of clinical events. However, aortic regurgitation is not often seen in young patients with quadricuspid aortic valve. Valvular regurgitation usually develops as a result of fibrous thickening with incomplete coaptation. With the unequal distribution of stress and abnormal leaflet coaptation, aortic regurgitation may occur later in life.

Aortic valve replacement for a quadricuspid valve is generally the treatment of choice for patients with valvular regurgitation. Only a few cases of aortic valve repair have been reported. Iglesias and associates ${ }^{1}$ reported the case of a patient with a type $\mathrm{B}$ quadricuspid aortic valve that was converted into a tricuspid valve by suturing together the commissure between the right coronary and accessory cusps. On the basis of that report, we performed aortic valve repair in our patient with intraoperative videoscopic evaluation. Because of adequate pressure on the aortic root in our method, a videoscope can evaluate the position of aortic valves accurately. There are some reports regarding the failure of valve repair. $^{2}$ Intraoperative videoscopic monitoring can reduce the incidence of improper valve repair procedures.

Embryologically, development of the aortic valve leaflets occurs immediately after the development of the coronary artery origins from the sinuses of Valsalva. It is therefore possible that these two groups of anomaly are embryologically related. Al-
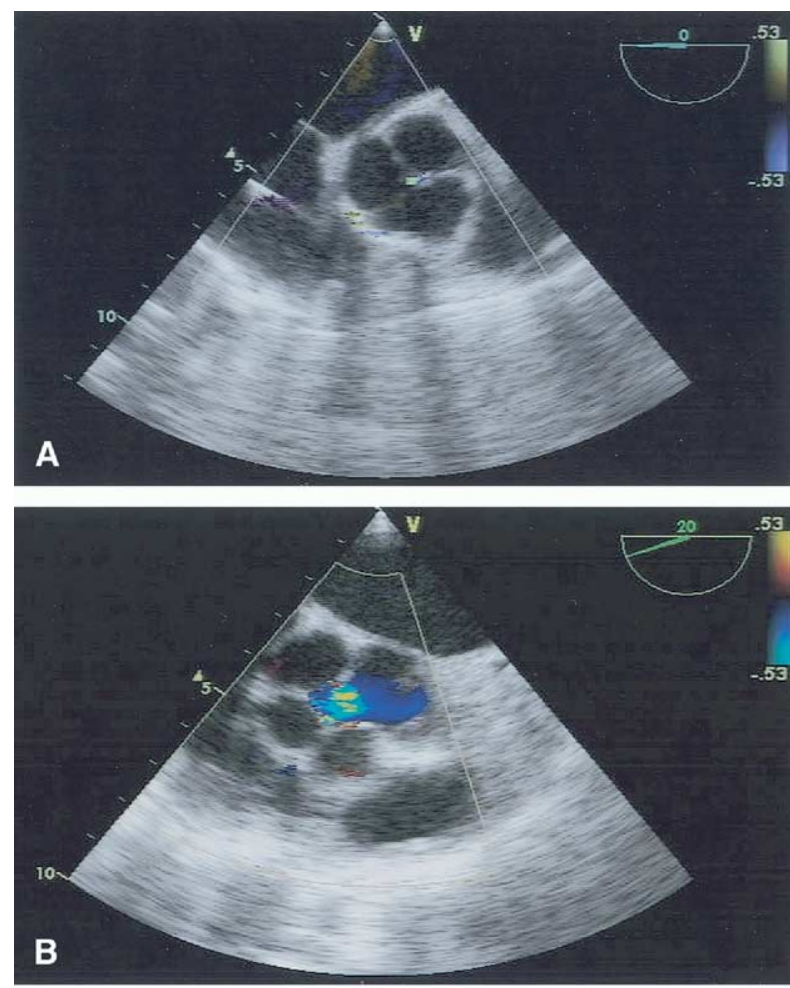

Figure 3. A, Postoperative transesophageal echocardiogram (short-axis view, diastolic phase). B, Preoperative transesophageal echocardiogram (short-axis view, diastolic phase).

though there was no evidence of coronary displacement in our patient, anomalies of coronary artery origin and distribution occur in about $30 \%$ of quadricuspid aortic valves. From the viewpoint of the surgeon, it is important to be aware of any displacement of the coronary ostium to prevent ostial obstruction at the time of valve replacement or repair.

\section{References}

1. Iglesias A, Oliver J, Munos JE, Nunez L. Quadricuspid aortic valve associated with fibromuscular subaortic stenosis and aortic regurgitation treated by conservative surgery. Chest. 1981;80:327-8.

2. Yotsumoto G, Iguro Y, Kinjo T, Matsumoto H, Matsuda H, Sakata R. Congenital quadricuspid aortic valve: report of nine surgical cases. Ann Thorac Cardiovasc Surg. 2003;9:134-7.

3. Hurwitz LE, Roberts WC. Quadricuspid semilunar valve. Am J Cardiol. 1973;31:623-6.

4. Feldman BJ, Khandhernia BK, Warnes CA, Seward JB, Taylar CL, Tajik AJ. Incidence, description and functional assessment of isolated quadricuspid aortic valves. Am J Cardiol. 1990;65:937-8.

5. Nakamura Y, Taniguti I, Saiki M, Morimoto K, Yamaga Y. Quadricuspid aortic valve associated with aortic stenosis and regurgitation. Jpn J Thorac Cardiovasc Surg. 2001;49:714-6. 